\title{
Microstructure-based analysis of fine metal mask cleaning in organic light emitting diode display manufacturing
}

\author{
Yong-Cheol Jeong ${ }^{1}$, Jun-Ho Yu ${ }^{1}$, Kwon-Yong Shin ${ }^{1}$, Sol Lee ${ }^{2}$, Chang-Ro Yoon ${ }^{2}$ and Sang-Ho Lee ${ }^{1 *}$ (D)
}

\begin{abstract}
This study proposes the unique method to analyze the cleanliness of the fine metal mask (FMM) used in OLED display manufacturing after FMM cleaning process. We developed a FMM-mimic microstructure as a substitute for the FMM, which can be used for the evaluation of cleaning efficiency. The FMM-mimic microstructure was fabricated using a combination of photolithography, reactive ion etching, anodic bonding and sand blasting processes. To demonstrate the proposed cleanliness analytical method, a $1.4 \mu \mathrm{m}$-thick Tris-(8-hydroxyquinoline) aluminum ( $\mathrm{Alq}_{3}$ ) film was deposited on the FMM-mimic microstructure as a contaminant by vacuum thermal evaporation. The $\mathrm{Alq}_{3}$-deposited FMM-mimic microstructure was cleaned by N-methyl-2-pyrrolidone (NMP) with changing cleaning time. We analyzed the residual contaminants on the FMM-mimic microstructure using a fluorescence microscope. The developed FMMmimic microstructure proves very convenient for inspecting the residual contaminant inside the gap through the transparent glass by general optical and fluorescence microscopy.
\end{abstract}

Keywords: Microstructure, Analysis, Fine metal mask, Cleaning, Organic light emitting diode, Display

\section{Introduction}

In the manufacture of flat panel displays for television screens, cell phone displays, computer monitors, and so on, organic electroluminescent displays (OLEDs) have attracted attention due to their large angle visibility, high brightness, wide range of working temperature, fast response time, high contrast and vivid color compared with traditional flat panel displays such as liquid crystal displays [1-4]. A typical OLED display structure consists of multi-organic layers such as an electron injection layer, an electron transport layer, a hole transport layer, and a hole injection layer which are sandwiched between a transparent indium-tin-oxide anode and a reflective metallic cathode. To form pixels emitting $\operatorname{red}(\mathrm{R})$, green $(\mathrm{G})$ and blue $(\mathrm{B})$ colors, pixels patterns are selectively deposited onto a thin film transistor (TFT) backplane panel with pixel bank array by evaporation

\footnotetext{
*Correspondence: sholee7@kitech.re.kr

1 Korea Institute of Industrial Technology, 143 Hanggaul-ro, Ansan-si,

Gyeonggi-do 15588, Republic of Korea

Full list of author information is available at the end of the article
}

of organic light emitting materials through a fine metal shadow mask (FMM) with tiny pixel-shaped apertures after highly precise alignment of the FMM to the TFT backplane [1,3-5].

The FMM is typically fabricated by laser welding of a thin metal sheet onto a stainless steel frame as the schematic of Fig. 1a shows. The thin metal sheet includes tiny openings formed by electroforming or micro photo etching of stainless steel or Invar (64\% Fe-36\% Ni alloy). The thin metal sheet of the FMM is only $30-200 \mu \mathrm{m}[6$, 7]. Due to its limited ductility and low thickness, it is extremely difficult to keep the FMM aligned and attached to the TFT back plane with high positional accuracy, so the thin metal sheet needs to be stretched and supported by a thick metal frame using laser welding. However, due to the technical limitation of FMM fabrication, a gap is created between the thin metal sheet and the stainless steel frames as shown in Fig. 1b which is an enlarged cross-sectional view of a portion surrounded by the circle on line $\mathrm{A}-\mathrm{A}^{\prime}$ in Fig. 1a $[8,9]$. 


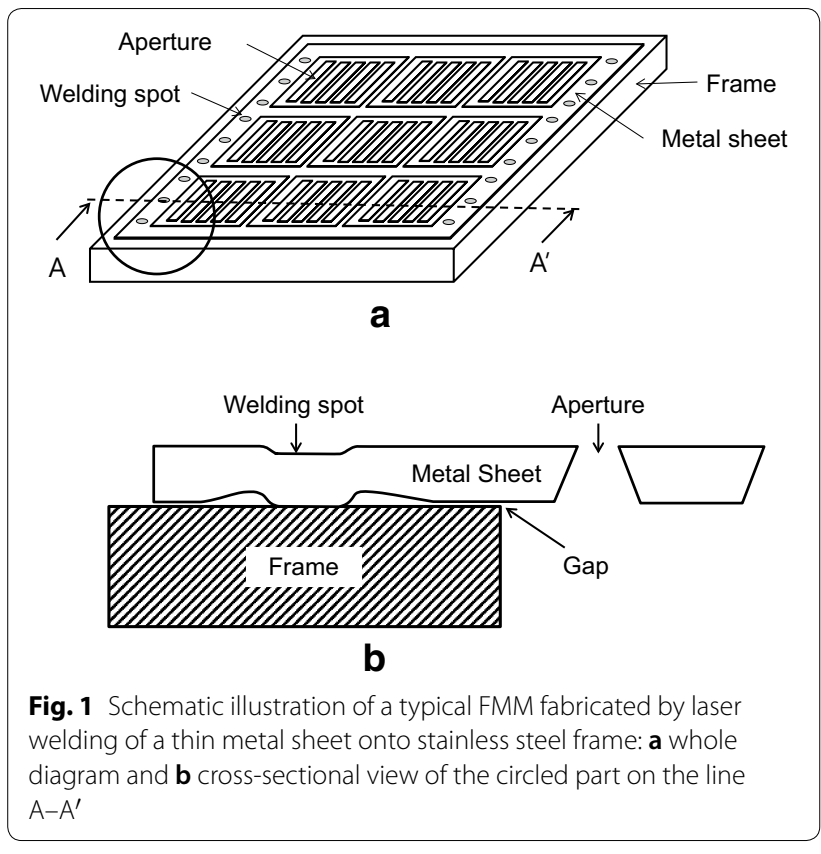

When an FMM is used repeatedly during the continuous manufacturing of a full color OLED, evaporated organic materials accumulate on the surface and interface of the FMM as well as inside the gap between the metal sheet and the stainless steel frame. The long time usage of the FMM causes the blocking of the tiny apertures and the distortion of the FMM, and eventually, the organic material cannot be accurately patterned to form an organic light emitting layer. Therefore, the FMM should be regularly cleaned to avoid patterning error caused by the accumulated materials, however, cleaning can lead to the damage of the FMM [5, 10, 11].

To find suitable cleaning conditions for minimizing FMM damage, it is important to analyze the efficiency of the FMM cleaning process. In general, the residual contaminant on the surface of an FMM can be analyzed by traditional analytical techniques such as VPD/ICP-MS, TXRF, XRF, AES, XPS and SIMS which are used to detect ionic and organic contamination on the wafer surfaces $[12,13]$. The particle contamination on the surface can also be measured by light-scattering-based surface scanners used for measuring particle in the efficiency evaluation of wafer cleaning systems $[12,14,15]$. However, it is impossible to investigate the residual contaminant inside the gap between the welded thin metal sheet and the frame because the metal sheet is opaque and the separation of the welded metal sheet is impossible until the last stage of FMM recycling. The residual contaminants inside the gap could take a longer time reaching the base vacuum pressure in the evaporation chamber for subsequent RGB patterning. Recently, Pyo et al. reported their research on chemical analysis of FMM cleaning process

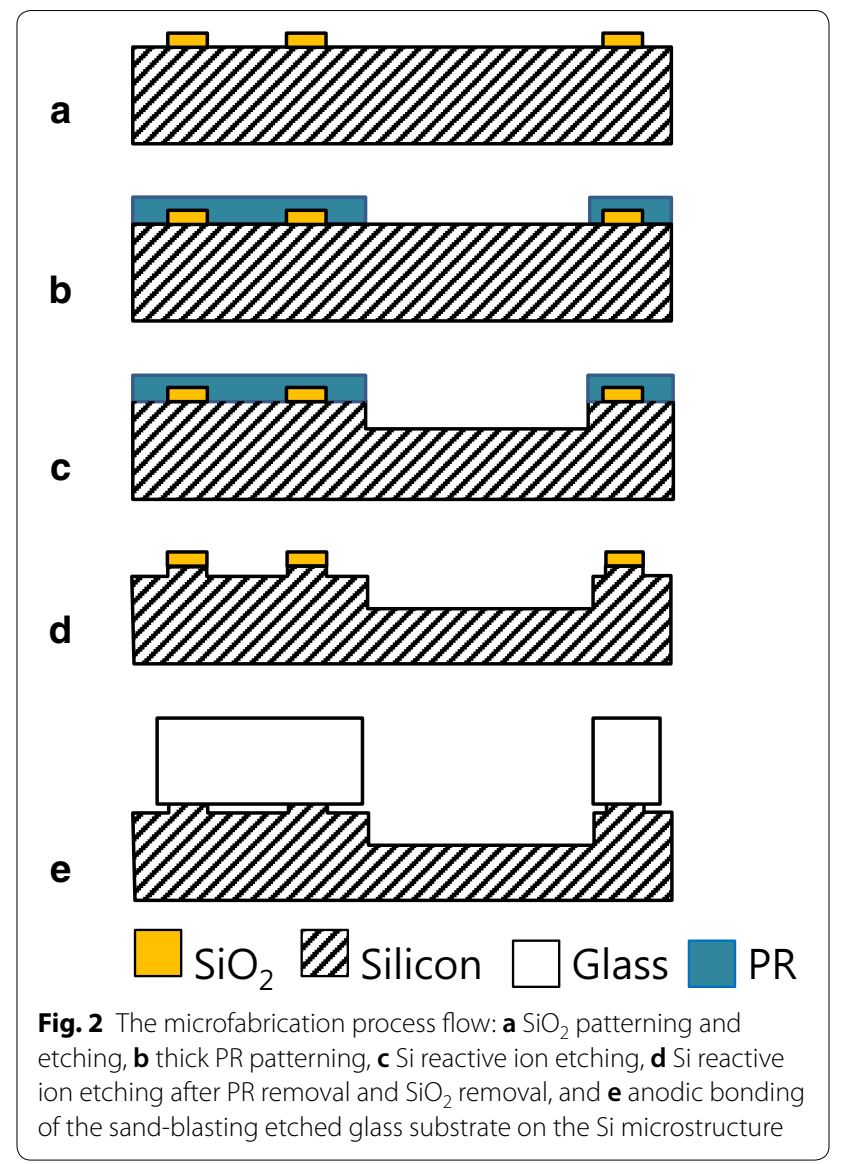

with confocal Ramen spectroscopy. They analyzed the cleaning solution used in FMM cleaning and made an analytical model to determine the concentration of residue in the cleaning solution [16].

Our study was aimed to find a unique method to analyze the residual contaminants inside the gap as well as on the surface of an FMM. Instead of an FMM, we developed a FMM-mimic microstructure that can be used for the evaluation of cleaning efficiency after FMM cleaning process. A $1.4 \mu \mathrm{m}$-thick Tris-(8-hydroxyquinoline) aluminum $\left(\mathrm{Alq}_{3}\right)$ film was deposited on the FMM-mimic microstructure as a contaminant by vacuum thermal evaporation. We analyzed the residual contaminants inside the gap using an optical and fluorescence microscope after the $\mathrm{Alq}_{3}$-deposited FMM-mimic microstructure was cleaned by $N$-methyl-2-pyrrolidone (NMP).

\section{Experimental methods}

To fabricate the FMM-mimic microstructure, we performed photolithography, reactive ion etching (RIE), anodic bonding and sand blasting processes. Figure 2 shows the microfabrication process flow: a $1 \mu \mathrm{m}$-thick $\mathrm{SiO}_{2}$ is first deposited on a silicon substrate using a PECVD (plasma enhanced chemical vapor deposition) 
a

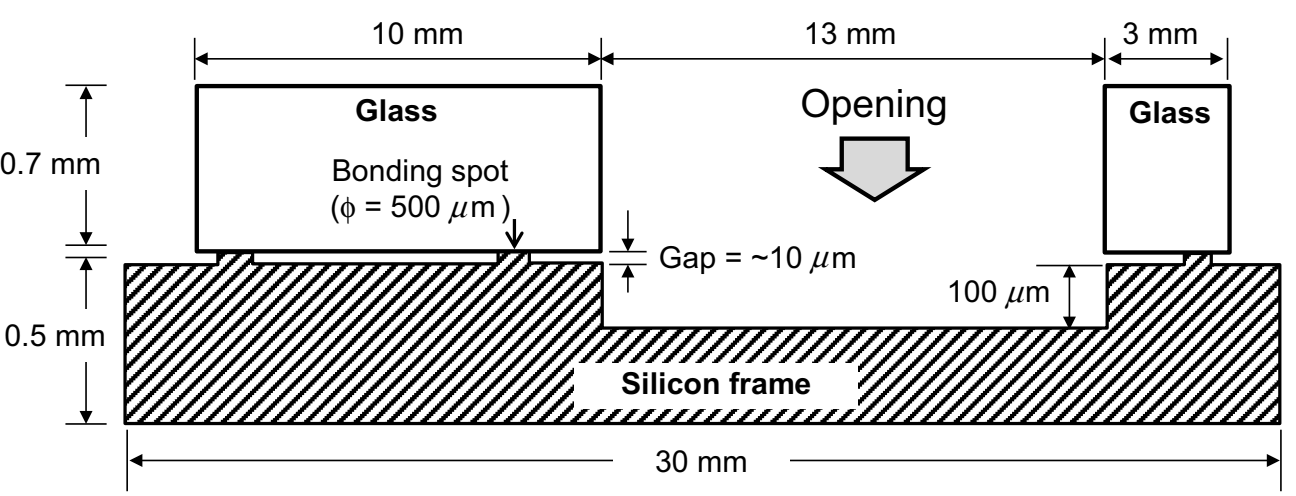

b

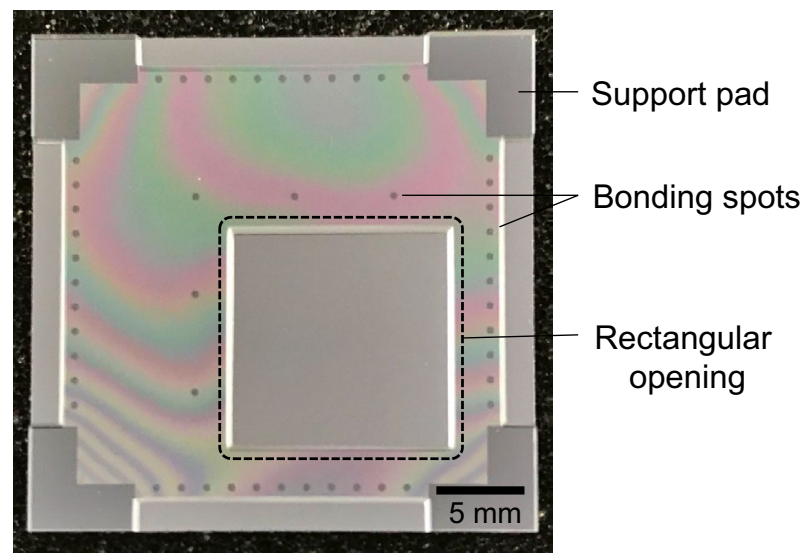

C

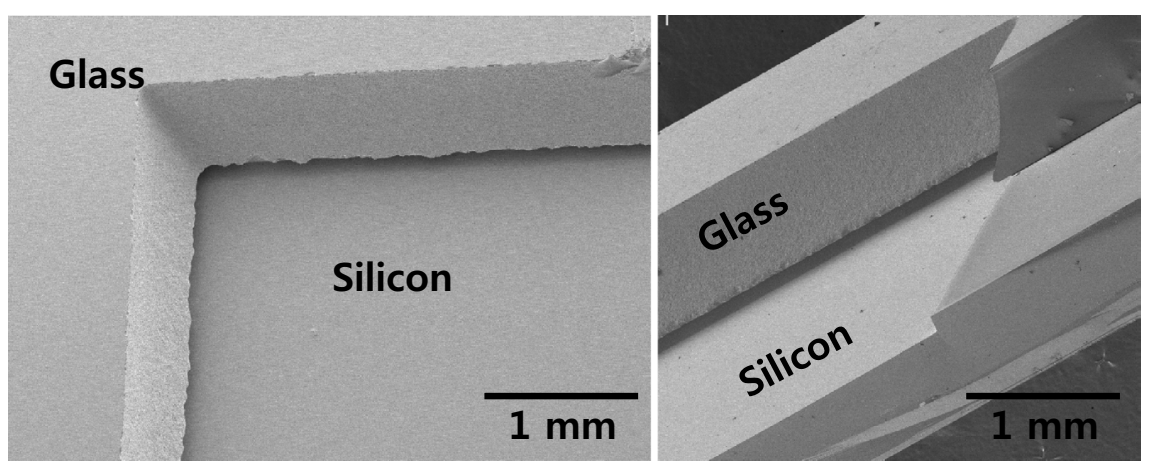

d

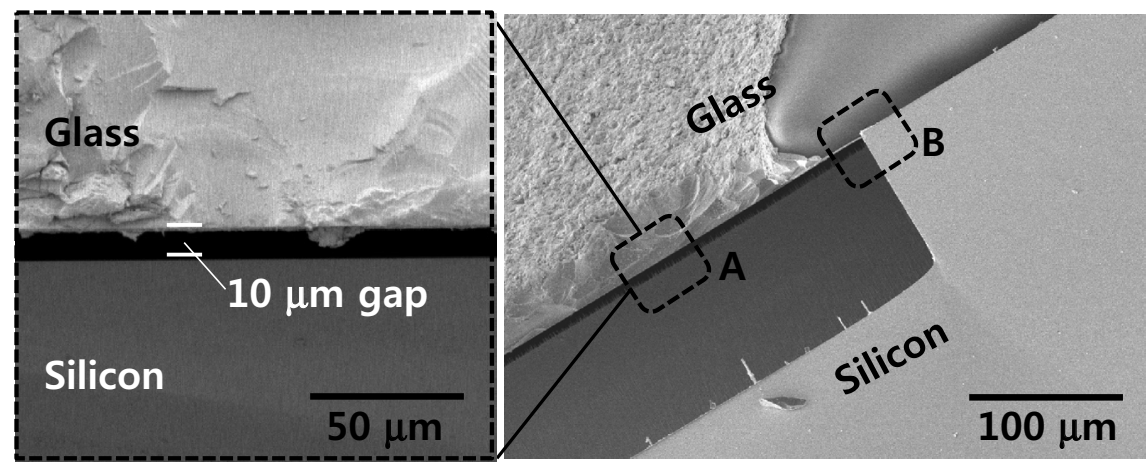


(See figure on previous page.)

Fig. 3 Microfabrication results: a cross-sectional scheme of the FMM-mimic microstructure, $\mathbf{b}$ picture of the fabricated FMM-mimic microstructure, c SEM images of the top and tilt view position at the corner of a rectangular opening, and $\mathbf{d}$ SEM images of the gap between a Si substrate and a glass substrate

system (NEXSO 7000 HDPECVD, NEXSO, Korea). $\mathrm{SiO}_{2}$ layer is etched as a circular shape to define bonding spots by a RIE system (RIE 80 plus, Oxford Instruments, UK) after patterning of a photoresist (PR) (AZ 5214E, MicroChemicals, Germany) (Fig. 2a). Then, the $15 \mu \mathrm{m}$-thick PR (AR-N 44000, Allresist, Germany) was patterned as an etch mask (Fig. 2b) and a $100 \mu$ m-deep rectangular cavity was formed by a Si deep RIE system (Rapier, SPTS, UK) (Fig. 2c). After removal of the thick PR, a second Si deep RIE was carried out to form $10 \mu \mathrm{m}$ high bonding spots, and the $\mathrm{SiO}_{2}$ layer was removed by a buffered oxide etchant (Fig. 2d). A glass substrate was etched by sand blasting with a dry film resist etch mask (MS7100, Mitsubishi Paper Mills, Japan) to form an opening that matched to the rectangular cavity. Finally, the glass substrate with the rectangular opening was aligned and bonded by an anodic bonding system (SB6, SUSS MicroTec, Germany) onto the silicon substrate with the bonding spots and the square cavity in a wafer level (Fig. 2e). The bonded sample was diced into $30 \mathrm{~mm} \times 30 \mathrm{~mm}$.

To test the feasibility of the proposed cleanliness analytical method, we deposited $1.4 \mu \mathrm{m}$-thick $\mathrm{Alq}_{3}$ film as a contaminant on the FMM-mimic microstructure by vacuum thermal evaporation. $N$-methyl-2-pyrrolidone (NMP) was used as a cleaning chemical and iso-propyl alcohol (IPA) and deionized (DI) water were used for rinsing after the NMP cleaning. We analyzed the residual contaminants with an optical and fluorescence microscope after nitrogen gas drying.

The photoluminescent images of the residual emitting materials were recorded by an objective lens coupled with a homemade reflective fluorescence microscope. The samples were excited through an objective lens (Edmund optics, $5 \times, 0.14 \mathrm{NA}$ ) by using collimated UV light emitting diodes (Thorlabs, $365 \mathrm{~nm}$ ). The emission from the sample was collected by the same objective lens and detected by a charge-coupled device.

\section{Results and discussion}

Figure 3a shows the cross-sectional scheme of the FMMmimic microstructure that imitated the gap structure of the metal sheet-welded part of an FMM (Fig. 1b). In this study, to imitate the thin metal sheet with numerous apertures, a large rectangular opening of $13 \mathrm{~mm} \times 13 \mathrm{~mm}$ was formed on the glass substrate instead of tiny apertures because sand blasting cannot form tiny apertures less than $\sim$ tens of micrometer on a $500 \mu \mathrm{m}$-thick glass substrate. Additionally, one large opening is a much more effective structure to make the evaporated $\mathrm{Alq}_{3}$ easily reaches the gap through the window. $10 \mu \mathrm{m}$-high bonding spots were designed to form $10 \mu \mathrm{m}$ gap between the glass substrate and the Si substrate after the bonding process. The spot's diameter was $500 \mu \mathrm{m}$. The dimensions on the spot diameter and gap were determined by referring to previous studies $[8,9,12]$.

The FMM-mimic microstructure was successfully fabricated by the proposed microfabrication process as the designed dimensions and as a result, Fig. $3 \mathrm{~b}$ shows a photograph of the fabricated FMM-mimic microstructure including a rectangular opening (center), bonding spots (perimeter) and support pads (corner). Figure 3c shows the SEM images of the top and tilt view positions at the corner of a square opening. We also confirmed that the gap was well maintained with $10 \mu \mathrm{m}$ between the glass substrate and the silicon structure without sink-down phenomenon as shown in the SEM images of Fig. 3d. The main advantage of the developed FMM-mimic microstructure is that we could easily inspect the contaminants inside the gap through the transparent glass by general optical and fluorescence microscopy. Also, during the optimization of the cleaning process, we could inspect the residual contaminant inside the gap without breakage of the sample while changing cleaning conditions.

To test the feasibility of the proposed analytical method using the FMM-mimic microstructure after FMM cleaning, we deposited a 1.4- $\mu \mathrm{m}$ thick $\mathrm{Alq}_{3}$ film on the FMMmimic microstructure by vacuum thermal evaporation. Then, the $\mathrm{Alq}_{3}$-deposited FMM-mimic microstructure was cleaned by NMP solution followed by cleaning with iso-propyl alcohol and deionized water. Figure 4a shows the FMM-mimic microstructure after $\mathrm{Alq}_{3}$ film deposition and Arabic numbers $(1,2,3)$ indicate observation regions after NMP cleaning. Figure $4 \mathrm{~b}$ shows the SEM images of the gap corresponding to view positions $A$ and $\mathrm{B}$ in Fig. $3 \mathrm{~d}$ after $\mathrm{Alq}_{3}$ film deposition. $\mathrm{Alq}_{3}$ was deposited the entrance of the gap, so the gap distance decreased

(See figure on next page.)

Fig. 4 Feasibility test of the cleanliness analytical method: a picture of FMM-mimic microstructure after $\mathrm{Alq}_{3}$ film deposition, b SEM images of the gap after $\mathrm{Alq}_{3}$ film deposition (before cleaning) and $\mathbf{c}-\mathbf{e}$ fluorescence microscopic images at the inspection regions $(1,2,3)$ for cleaning time (Arabic numbers indicated the inspection regions, respectively) 
a

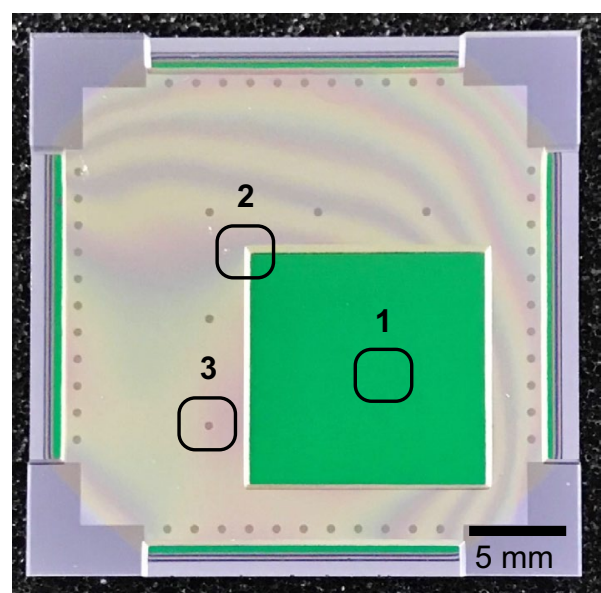

1.4 $\mu \mathrm{m}$-thick $\mathrm{Alq}_{3}$ deposited FMM-mimic microstructure

b
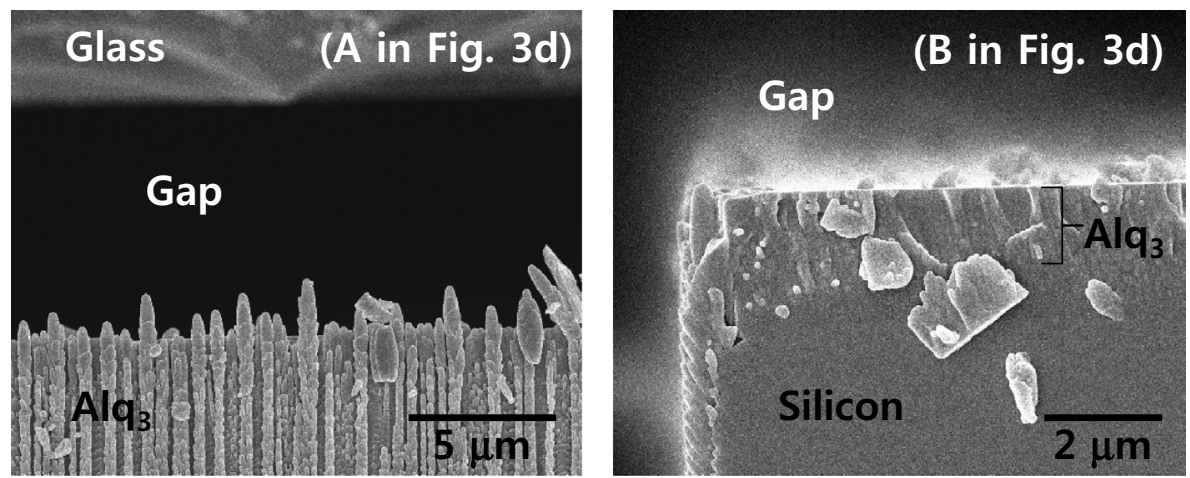

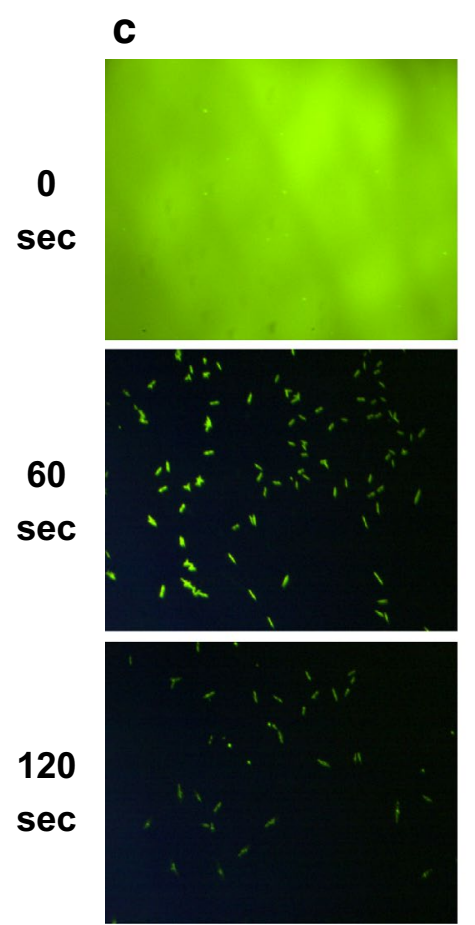

Region 1 d
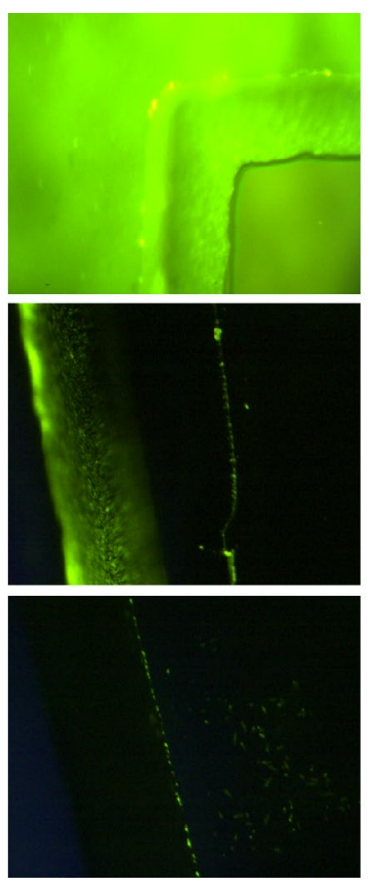

Region 2 e
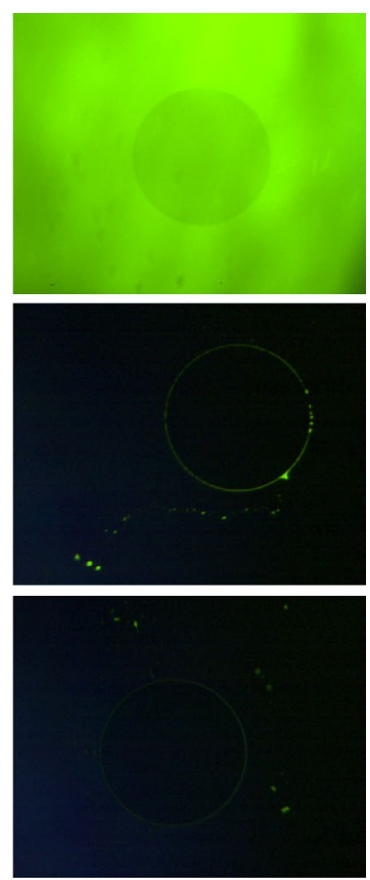

Region 3 
from 10 to $\sim 8 \mu \mathrm{m}$. To analyze the degree of cleaning of the FMM-mimic microstructure, we monitored $\mathrm{Alg}_{3}$-coated samples (Fig. 4a) by our homemade fluorescence microscope regarding cleaning time. Figure $4 \mathrm{c}-\mathrm{e}$ shows green-colored images clearly in all areas indicating a uniformly deposited $\mathrm{Alq}_{3}$ thin layer on the FMMmimic substrate before NMP solvent cleaning (0 s). After dipping the sample in the NMP solvent for $60 \mathrm{~s}$, the thin layer of light-emitting material was almost washed, whereas residual aggregates of $\mathrm{Alq}_{3}$ were still observed. Meanwhile, the relatively bright green-light emission is detected on the edge line of the sand blasted surface in the upper glass substrate as shown in Fig. 4d. This can be explained in that the $\mathrm{Alq}_{3}$ molecules are bound to the surface more tightly due to the considerable roughness of the edge surface. Moreover, after NMP solvent cleaning for $60 \mathrm{~s}$, Fig. 4e shows a circular string of green-light emission. It is conjectured that the dissolved $\mathrm{Alq}_{3}$ in NMP flowed into the gap (Fig. 3d) during the cleaning process and seemed to be trapped around the gap spacer (circular bonding spot) because of high surface tension. With further dipping in NMP solvent for $120 \mathrm{~s}$, the light intensity of the green-visible emission decreased, resulting in a lower amount of $\mathrm{Alq}_{3}$ residuals.

\section{Conclusions}

This study successfully demonstrated the FMM-mimic microstructure based analysis method to evaluate the cleanliness of an FMM after the wet cleaning process used in OLED manufacturing. The FMM-mimic microstructure was developed using a micromachining process, which included photolithography, RIE, anodic bonding and sand blasting processes. To mimic the FMM corner portion, the FMM mimic microstructure was designed to include a rectangular opening, bonding spots, and support pads, and a gap of a $10 \mu \mathrm{m}$ was formed between the glass substrate and the silicon structure. For the feasibility test of the proposed cleanliness analysis method, a $1.4 \mu$ m-thick $\mathrm{Alq}_{3}$ film was deposited on the FMM-mimic microstructure as a contaminant. With varying NMP cleaning time, we effectively analyzed the residual contaminants inside the gap as well as the surface of the FMM-mimic microstructures by the fluorescence microscope. The FMM-mimic microstructure is particularly to monitor the $\mathrm{Alq}_{3}$ contaminant trapped around the bonding spots, which was the main research objective to be solved in this study.

\footnotetext{
Authors' contributions

$\mathrm{S}-\mathrm{HL}$ proposed the main concept for the cleanliness analytical method, designed the FMM-mimic microstructure, and wrote the overall manuscript. Y-CJ performed the cleanliness analysis and assisted in writing the manuscript. $\mathrm{J}-\mathrm{HY}$ and $\mathrm{K}-\mathrm{YS}$ performed the device fabrication. SL performed the cleaning experiments. C-RY supervised the research and reviewed the manuscript. All authors read and approved the final manuscript.
}

\section{Author details}

${ }^{1}$ Korea Institute of Industrial Technology, 143 Hanggaul-ro, Ansan-si, Gyeonggi-do 15588, Republic of Korea. ${ }^{2}$ Deviceeng, 169 Eumbong-ro, Eumbong-myeon, Asan-si, Chungcheongnam-do 31415, Republic of Korea.

\section{Competing interests}

The authors declare that they have no competing interests.

\section{Availability of data and materials}

The datasets supporting the conclusions of this article are included within the article.

\section{Ethics approval and consent to participate}

Not applicable.

\section{Funding}

This work was supported from Advanced Technology Center Program of MOTIE (Ministry of Trade, Industry and Energy) of Republic of Korea (Project Number: 10062356)

\section{Publisher's Note}

Springer Nature remains neutral with regard to jurisdictional claims in published maps and institutional affiliations.

Received: 19 September 2018 Accepted: 14 January 2019

Published online: 19 January 2019

\section{References}

1. Chen S, Kwok H-S (2012) Full color organic electroluminescent display with shared blue light-emitting layer for reducing one fine metal shadow mask. Org Electron 13:31-35. https://doi.org/10.1016/j.orgel.2011.10.002

2. Jiang Y, Lian J, Chen S, Kwok H-S (2013) Fabrication of color tunable organic light-emitting diodes by an alignment free mask patterning method. Org Electron 14:2001-2003. https://doi.org/10.1016/j.orgel .2013.04.040

3. Lih J-J, Chao C-I, Lee C-C (2007) Novel pixel design for high-resolution AMOLED displays with a shadow mask. J. SID 15:3-7. https://doi. org/10.1889/1.2451539

4. Shin HY, Suh MC (2014) A study on full color organic light emitting diodes with blue common layer under the patterned emission layer. Org Electron 15:2932-2941. https://doi.org/10.1016/j.orgel.2014.08.033

5. Kim TJ (2014) Method and apparatus for cleaning organic materials. US Patent 9,733,561 B2, Aug. 15, 2017

6. Nagayama T, Yamamoto T, Nakamura T, Mizutani Y (2013) Fabrication of low CTE metal masks by the Invar Fe-Ni alloy electroforming process for large and fine pitch OLED displays. ECS Trans 50:117-122

7. Heo J, Min H, Lee M (2015) Laser micromachining of permalloy for fine metal mask. Int J Precision Eng Manuf Green Technol 2:225-230. https:// doi.org/10.1007/s40684-015-0026-7

8. Shigemura K (2009) Deposition mask frame assembly, method of fabricating the same, and method of fabricating organic electroluminescent device using the deposition mask frame assembly. US Patent 7,53,7798 B2, May 26, 2009

9. Kitazume E Metal mask structure and method for manufacturing thereof. US Patent 6,893,709 B2, May 17, 2005

10. Du et al (2015) Type of fine metal mask (FFM) used in OLED production and the method of manufacturing it US Patent 9,188,856 B2, Nov. 17, 2015

11. Lee et al (2015) Method and apparatus for cleaning organic deposition materials. US Patent 9,174,250 B2, Nov. 3, 2015

12. Baltzinger $J L$, Delahaye $B(2010)$ Contamination monitoring and analysis in semiconductor manufacturing. In: Jan G (ed) Semiconductor technologies. IntechOpen, UK, pp 57-78. https://doi.org/10.5772/8561 
13. Samantha HT, Ning C, Shi L, Kefei W (2003) Contamination-free manufacturing: tool component, qualification, verification and correlation with wafers. AIP Conf Proc 683:289-293. https://doi.org/10.1063/1.1622484

14. Lilienfeld $P$ (1986) Optical detection of particle contamination on surfaces: a review. Aerosol Sci Technol 5:145-165. https://doi. org/10.1080/02786828608959085

15. Huff HR, Goodall RK, Williams E, Woo K-S, Liu YH, Warner T, Hirleman D, Gildersleeve K, Bullis WM, Scheer BW, Stover J (1997) Measurement of silicon particles by laser surface scanning and angle-resolved light scattering. J Electrochem Soc 144:243-250. https://doi. org/10.1149/1.1837392

16. Kwon SH, Cui Y, Choi E, Kim A, Chae SJ, Pyo SG (2015) Chemical analysis of patterned mask cleaning in organic light emitting diode fabrication with Raman spectroscopy. Appl Spectrosc Rev 50:557-564. https://doi. org/10.1080/05704928.2015.1040923

\section{Submit your manuscript to a SpringerOpen ${ }^{\circ}$ journal and benefit from:}

- Convenient online submission

- Rigorous peer review

- Open access: articles freely available online

- High visibility within the field

- Retaining the copyright to your article

Submit your next manuscript at $\boldsymbol{s p r i n g e r o p e n . c o m ~}$ 